\title{
SELL expression associates with survival in triple negative breast cancer.
}

Shahan Mamoor, $\mathrm{MS}^{1}$

${ }^{1}$ shahanmamoor@gmail.com

East Islip, NY USA

We mined published microarray data (1) to understand the most significant gene expression differences in the tumors of triple negative breast cancer patients based on survival at time of analysis: dead or alive. We observed significant transcriptome-wide differential expression of selectin L, encoded by $S E L L$, when comparing the primary tumors of triple negative breast cancer patients dead or alive. Importantly, SELL expression was significantly correlated with overall survival in basal subtype breast cancer, a molecular subtype sharing significant overlap with triple negative breast cancer. SELL may be of relevance as a biomarker or as a molecule of interest in understanding the etiology or progression of triple negative breast cancer.

Keywords: triple negative breast cancer, TNBC, systems biology of breast cancer, targeted therapeutics in breast cancer, SELL, selectin L. 
Triple negative breast cancer is defined by lack of expression of the receptors for the hormones progesterone and estrogen (PR negative and ER negative), as well as for the human epidermal growth factor receptor 2 (HER2 negative) $(2,3)$. Though not completely identical, triple negative breast cancer shares significant overlap with the basal or basal-like molecular subtype of human breast cancer (3). TNBC is more frequently diagnosed in women of African descent (black women) for reasons not understood, and their disease course is similarly more aggressive than in white women with triple negative breast cancer $(2,3)$. To understand the transcriptional biology of triple negative breast cancer in an unbiased fashion and at the systems level, we mined published microarray data paired with patient survival data (1), performing comparative transcriptome analysis based on survival at time of data collection: dead or alive. We present here a finding from the preliminary results of this analysis.

\section{Methods}

We used dataset GSE142102 (1) for this differential gene expression analysis in conjunction with GEO2R. GSE142102 was generated using Affymetrix Human Gene 2.1 ST Array technology; in this analysis, we used data from $n=87$ tumors from TNBC patients that were dead at time of analysis and $n=123$ tumors from TNBC patients that were alive at time of analysis. The Benjamini and Hochberg method of $p$-value adjustment was used for ranking of differential expression but raw $p$-values were used to assess statistical significance of global differential expression. Log-transformation of data was auto-detected, and the NCBI generated category of platform annotation was used. A statistical test was performed to evaluate whether SELL expression was significantly different between the primary tumors of TNBC patients that were dead or alive using a two-tailed t-test. For Kaplan-Meier survival analysis, we used the Kaplan-Meier plotter tool (4) for correlation of SELL mRNA expression levels with overall survival (OS) in $n=404$ patients with basal subtype breast cancer.

\section{Results}

We performed comparative transcriptome analysis using published microarray data (1) to understand in an unbiased fashion the most significant gene expression differences in the primary tumors of patients with triple negative breast cancer based on survival outcomes.

$\underline{\text { SELL is differentially expressed in triple negative breast cancer and negatively correlates with survival. }}$

When comparing primary tumors of 87 patients with triple negative breast cancer, alive at time of data collection and 123 TNBC patients, dead at time of data collection, we identified the selectin L, SELL, as among the most significant transcriptional differences based on survival in human triple negative breast cancer. When ranking each of the transcripts whose expression was measured by microarray based on significance of difference in expression between the tumors of patients dead and alive, SELL ranked 226 out of 53617 total transcripts, equivalent to $99.6 \%$ differential expression (Chart 1). SELL differential expression in the tumors of TNBC patients based on survival outcomes was statistically significant (Figure $1 ; p=0.00559417)$. 
SELL is expressed at significantly higher levels in the breast tumors of TNBC patients when comparing based on survival.

We obtained exact mRNA expression levels for SELL from breast tumors of TNBC patients dead and alive to understand the magnitude and direction of SELL expression change based on survival outcomes. SELL was expressed at higher levels in tumors of TNBC patients alive as compared to that of TNBC patients dead (Figure 1). Increased expression of SELL in primary breast tumors was statistically significant (Figure 1: $p=0.0046$ ). SELL was expressed at $-0.19 \pm 0.87$ arbitrary units (AU) in the primary tumors of TNBC patients dead, while it was expressed at $0.20 \pm 1.09$ AU in the primary tumors of TNBC patients alive.

\section{$\underline{\text { SELL expression correlates with overall survival in basal subtype human breast cancer. }}$}

We referenced a bioinformatics tool containing Kaplan-Meier survival data of human breast cancer patients to understand more thoroughly whether a relationship existed between SELL primary tumor expression and patient survival in human breast cancer. We found that SELL expression was positively and significantly correlated with overall survival in patients with basal or basal-like human breast cancer, a breast cancer molecular subtype which shares significant overlap with triple negative breast cancer (Figure 2; log rank $p$-value: 5.2 e-05 for overall survival, hazard ratio: 0.45 (0.3-0.67) (Fig. 2)). SELL mRNA levels were a positive prognostic indicator in basal subtype breast cancer patients. Median OS was 35.53 months for basal subtype patients with low tumor expression of SELL as compared to 115 months for basal subtype patients with high tumor expression of SELL.

Thus, we found through unbiased comparative transcriptome analysis that SELL was among the genes whose expression was most significantly different in the primary tumors of triple negative breast cancer patients alive when compared to that of TNBC patients dead, that SELL was expressed at significantly higher levels in the primary tumors of TNBC patients alive as compared to TNBC patients dead, and that SELL expression was positively correlated with overall survival in the basal or basal-like molecular subtype.

\section{Discussion}

It is not understood why women of African descent (black women) are diagnosed with triple negative breast cancer more frequently than white women, and why this disease is more aggressive in black women. SELL may be of relevance as a biomarker in identifying patients who are more likely to survive, perhaps as part of a panel of genes used for prognostic stratification, and the molecule itself may be important to the biology of triple negative breast cancer etiology or more likely its progression. Blind systems-level analyses, like this, can help shed light on the fundamental molecular characteristics of a disease whose racial propensities demand further explanation. 


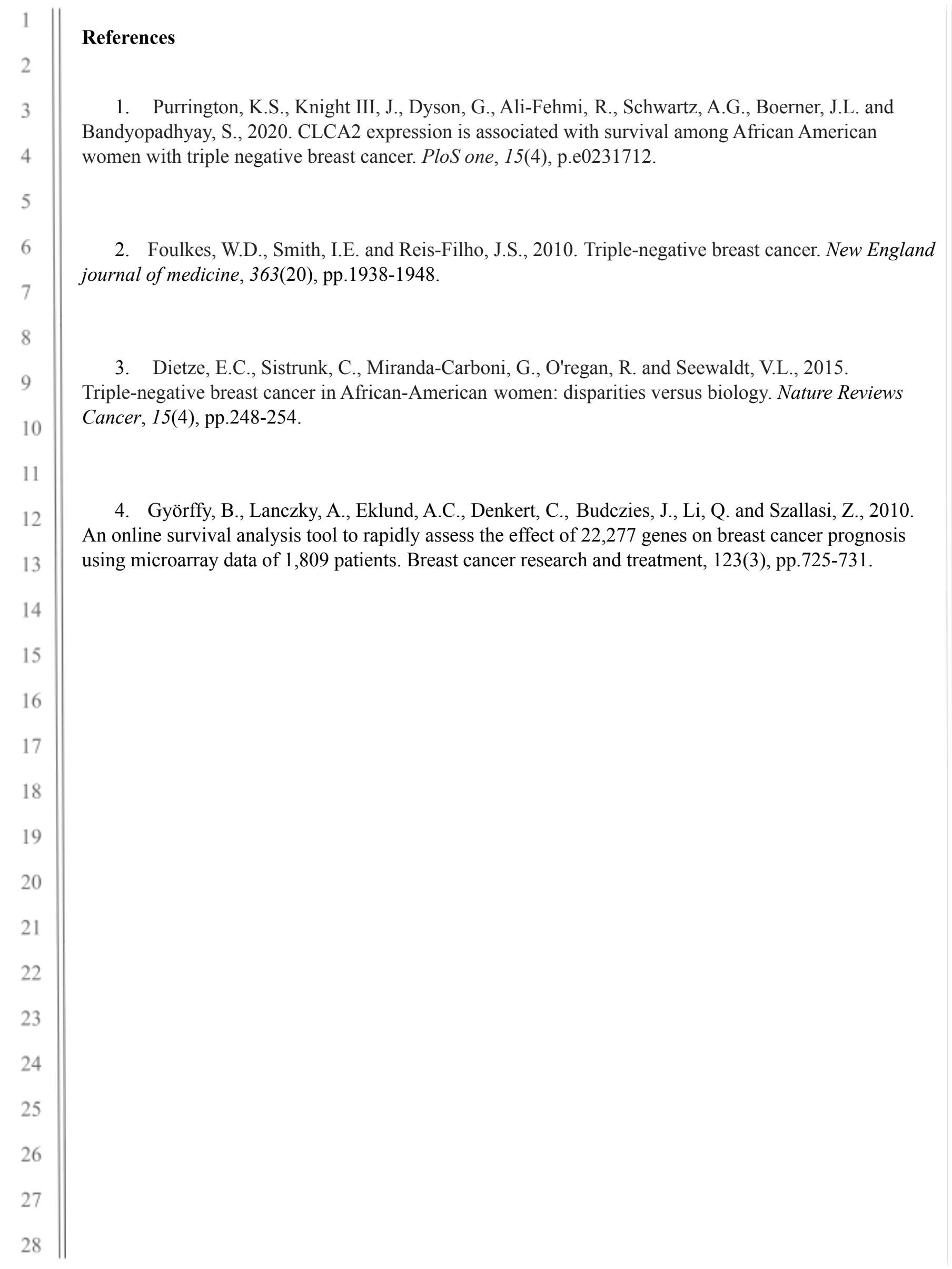




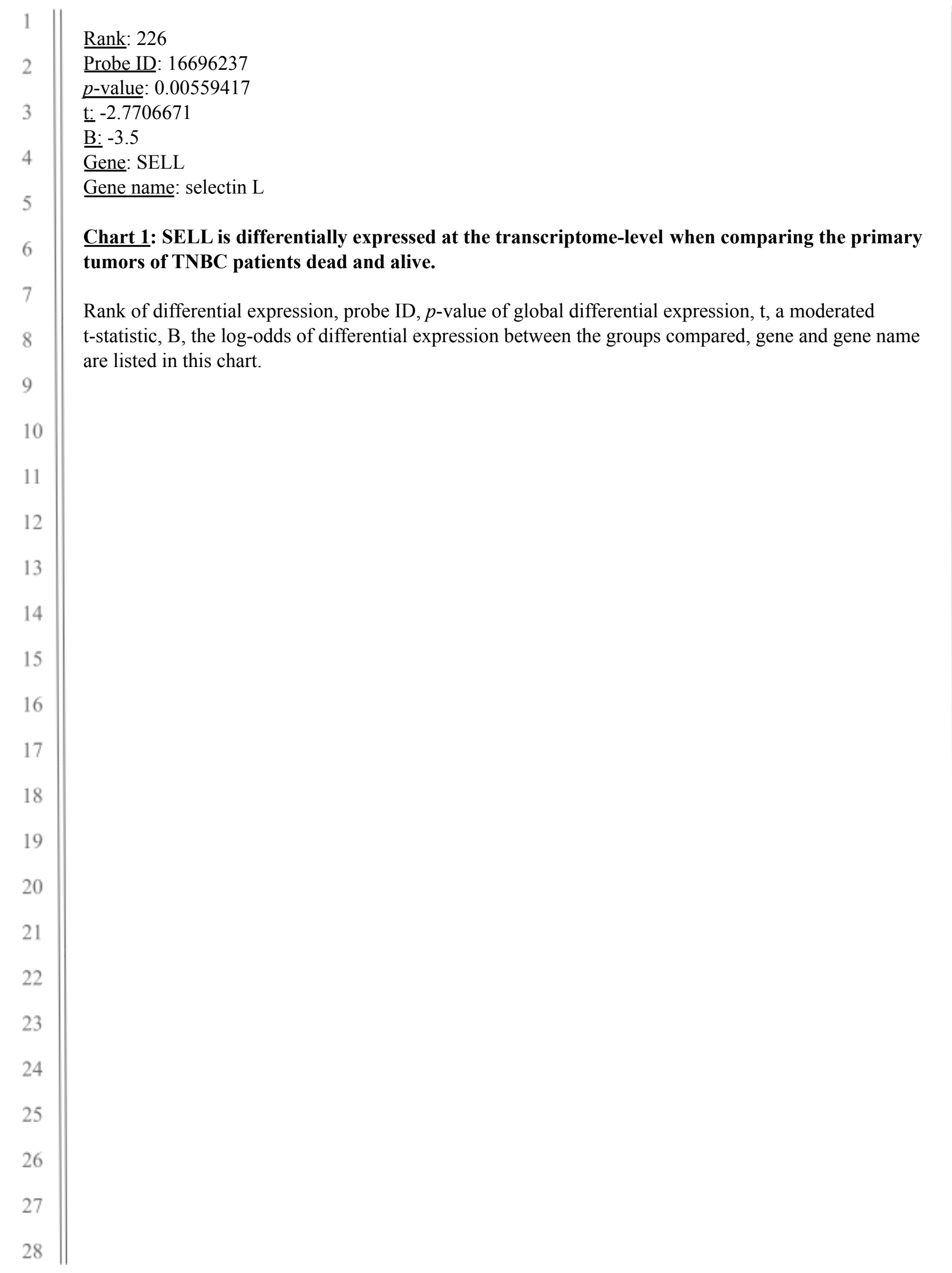




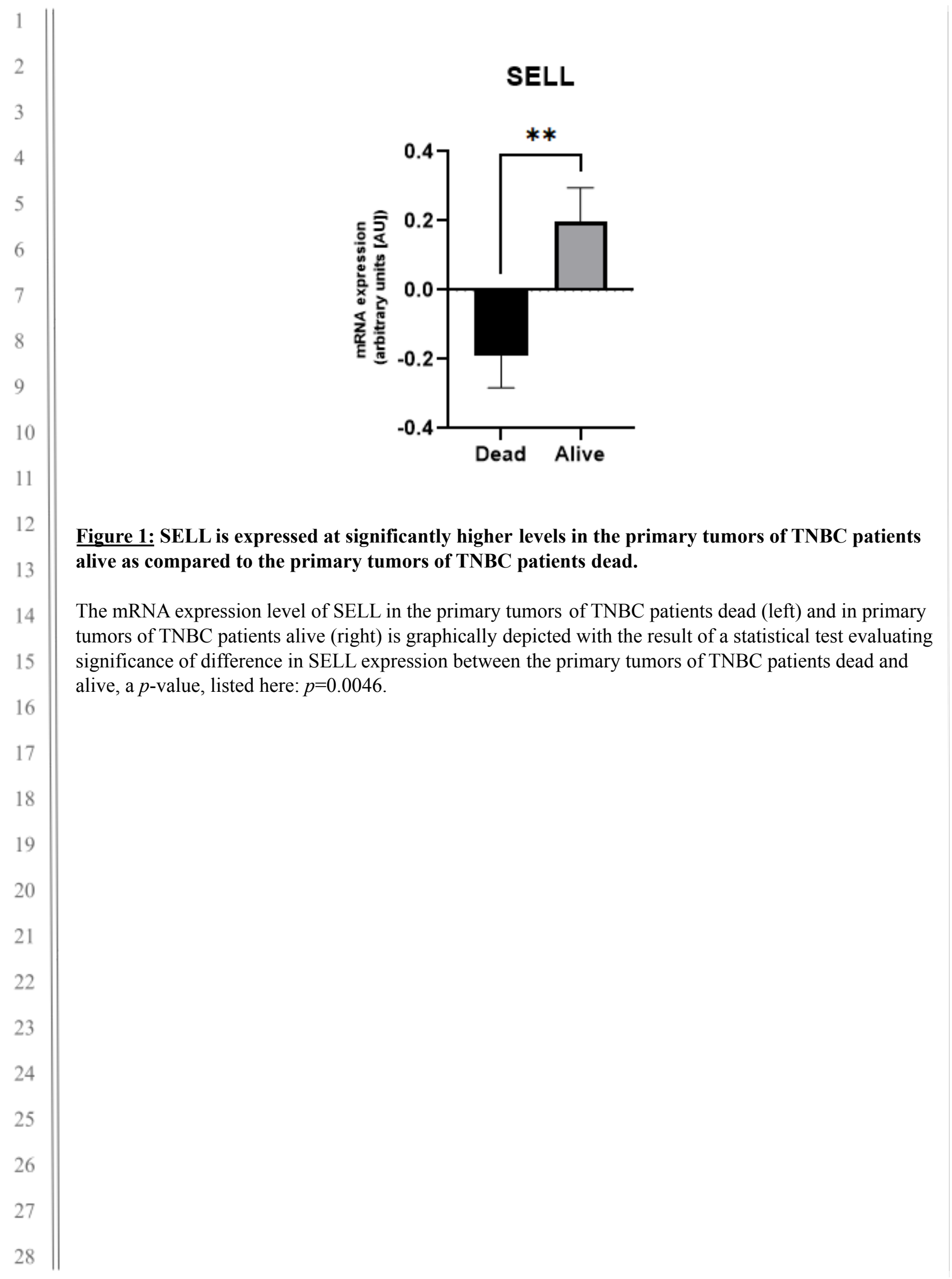




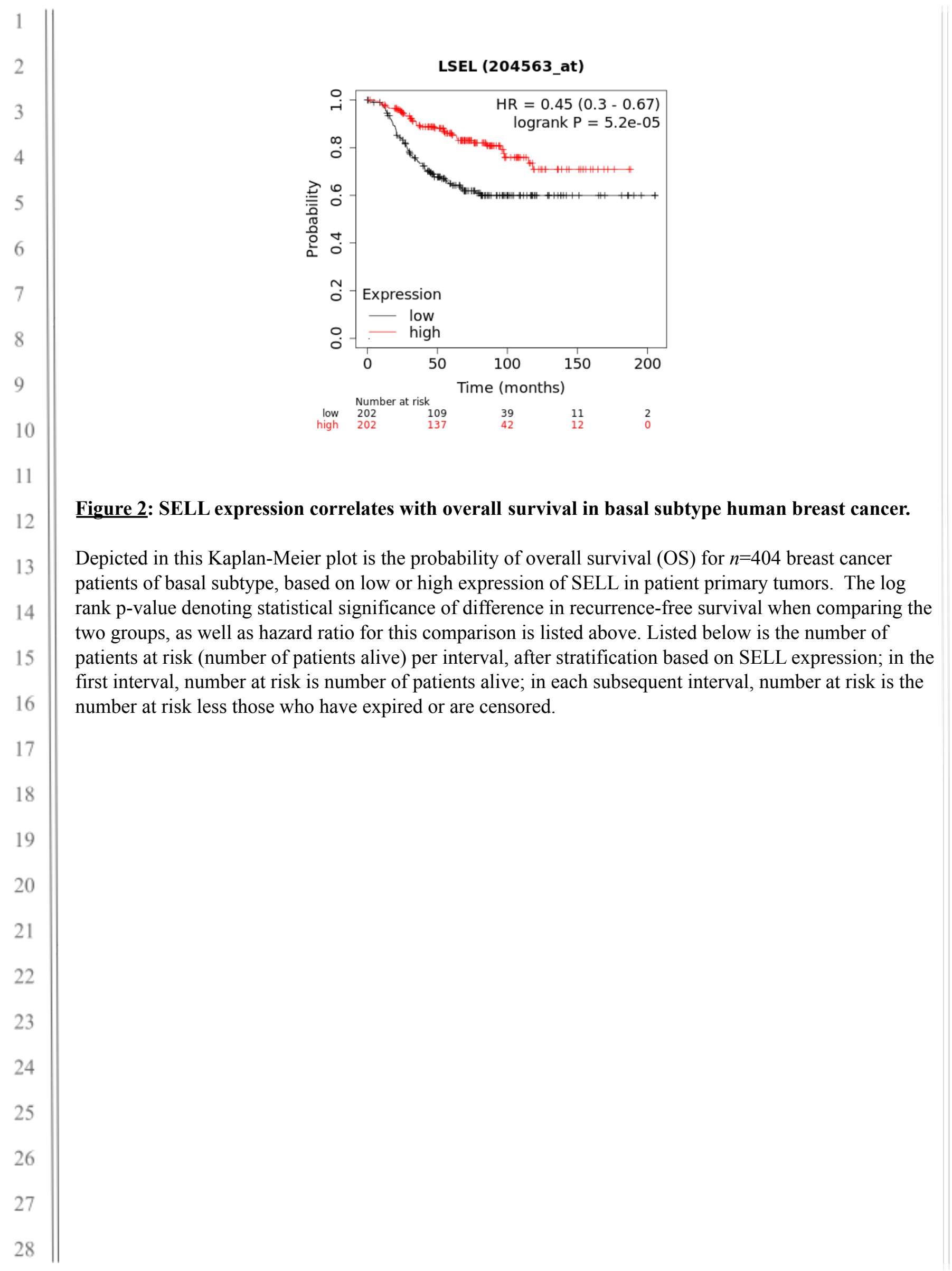


Low SELL expression: 35.53 months

High SELL expression: 115 months

Chart 2: Median overall survival is superior in basal subtype breast cancer patients with high primary tumor expression of SELL.

The median OS (overall survival) of basal subtype breast cancer patients, with low primary tumor expression of SELL and high primary tumor expression of SELL is listed in this chart. 